\section{Numerical modeling of vertical axis wind turbine with air guides}

\author{
Krzysztof Kołodziejczyk, Radosław Ptak, Jakub Korman \\ AGH University of Science and Technology, Poland
}

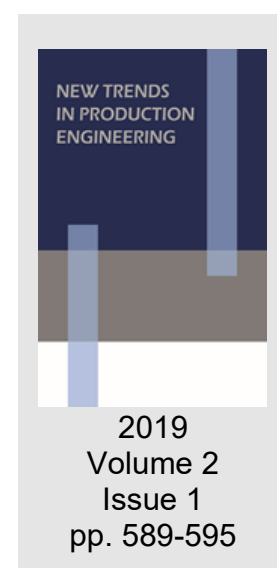

Date of submission to the Editor: 05/2019

Date of acceptance by the Editor: 08/2019

\title{
INTRODUCTION
}

The continuous growth in energy demand, with the simultaneous need to reduce its production from fossil fuels, makes it necessary to look for new solutions (Hudy et al., 2018, Turlej and Banaś, 2017). Solutions allowing for obtaining more energy from renewable resources (Hudy et al., 2019). One of the methods to reduce the use of natural resources is to improve the efficiency of energy conversion from renewable resources by introducing new solutions or optimizing existing ones (Rahman et al., 2018, Kacprzak and Sobczak, 2013). Harvesting wind energy is one of the first methods for obtaining energy from renewable sources. Over the last century, the use of this energy source has experienced a renaissance. Very intensive pro-ecological activities, as well as active policy of highly developed countries, including to limit the production of carbon dioxide, resulted in the extremely wide use of renewable technologies, including the use of wind energy. Intensive use of wind energy resources, through the construction of wind farms consisting mainly of wind turbines with horizontal axis (high power), next to a positive effect in the form of obtaining "green energy", causes locally negative impact on the environment, among others by generating noise, introducing changes in the landscape, causing a negative effect on birds resulted in negative public perception. The very dynamic development of wind energy resources through the construction of wind turbines with a horizontal axis of rotation, as a direct result of formal and legal restrictions, has been significantly limited. Therefore, it seems to be more important to search for solutions that allow harvesting wind energy with reduced negative impact on the environment. Searching for solutions that could be used on a smaller scale as local energy sources. The solution may be a wind turbine with a vertical axis. Such constructions, in addition to very important defects such as low efficiency and low rotational speeds (Fujisawa and Gotoh, 1994), have a number of advantages (Spera, 2009, Wagner and Mathur, 2009) among others:

- low noise emission level,

- independence of wind direction, 
- high starting torque is possible.

There are two distinctive types of vertical turbines such as Savonius and Darius, on the basis of which a number of modified constructional solutions were made. The construction, which has not been investigated to a wider extent yet, is the solution of a vertical axis windmill with air guides. This is on the one hand an attractive solution, because it allows to shape inflow into the rotor blades, and on the other, it is controversial because it significantly increases the complexity of the structure, and consequently raises its construction cost and in effect reduces the attractiveness of the solution. Nevertheless, this solution seems to be attractive enough to analyze the potential of such a construction.

\section{METHODOLOGY OF RESEARCH}

The publication presents a numerical analysis of the flow and operation of a wind turbine with a vertical axis of rotation, including the determination of the torque and power that can be obtained. The subject of the analysis has been a structural solution consisting of two elements: a rotor and an air guides ring. The structural solution of a windmill with a vertical axis, presented in Figure 1, has been limited to one level from a multi-level system. In the adopted system, the windmill can be built from a series of individual layers arranged one on the other vertically.

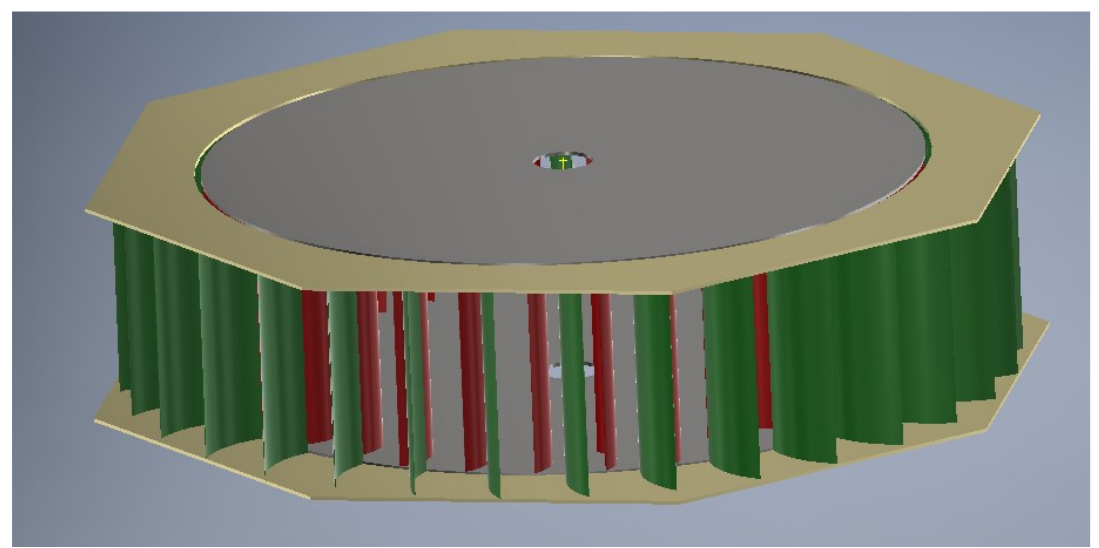

a)

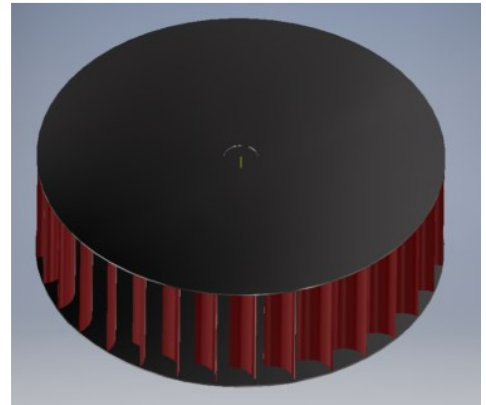

b)

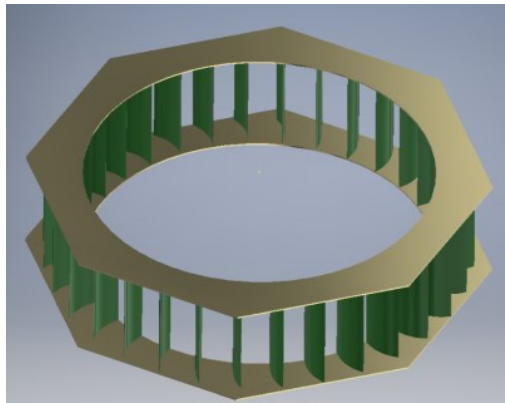

Fig. 1 Design solution to the vertical axis wind turbine a) rotor, b) air guides

Analyzed structural solution consists of a rotor with a diameter of $2.04 \mathrm{~m}$ and a height of $0.594 \mathrm{~m}$. It is a self-supporting structure consisting of two plates limiting the air flow space and 32 blades, which at the same time are connecting element between the top and bottom plates. Guiding ring is build in an analogous way. It 
consists of two limiting plates, top and bottom, which are connected with air guides.

For the presented construction, a numerical flow analysis was performed using the Ansys CFX software package. The analysis was carried out assuming a constant air flow velocity through the windmill of $4 \mathrm{~m} / \mathrm{s}$. This is the speed that can be assumed as dominant at the height of several to a dozen or so meters above ground level in Poland. Air at a temperature of $25^{\circ} \mathrm{C}$ was assumed as the medium flowing through the system. In order to perform the analysis of the work of a single-degree windmill, a computational domain was created, made as a cube with dimensions of $12000 \times 8000 \times 609 \mathrm{~mm}$ with a centrally located windmill. The computing space has been divided by a grid consisting of 3671 437 hexahedron elements (Figure 2).

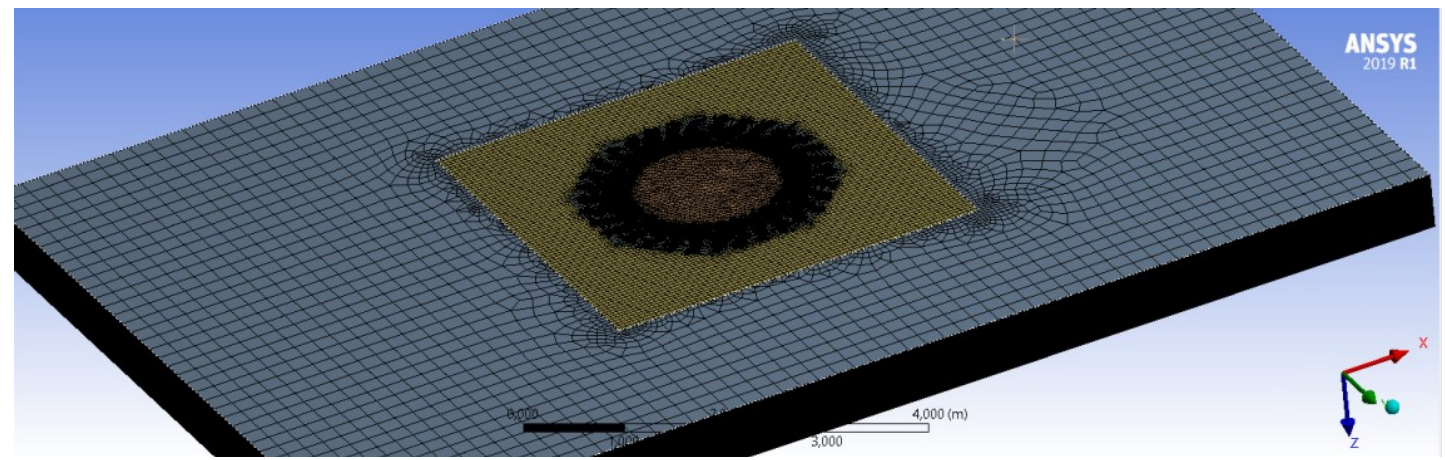

Fig. 2 Model divided with hexahedron grid

Constant in time analysis was conducted, with assumed lack of compressibility of the flowing factor. In conducted analysis, a constant rotor rotation speed of $15 \mathrm{rpm}$ was assumed. This speed was determined coarsely on the basis of literature based data on the efficiency and the specific speed. Because this type of data for the studied geometry is not yet defined, the coarse values have been determined as for the Savonius turbine for which the maximum efficiency values are at the level of $25 \%$. This is a big simplification in the analysis, but at this stage without laboratory verification, this method is sufficient. The conducted analysis took into account the turbulence of the flow, in the analysis the Shear Stress Transport (SST) model was adopted. This model was used in this type of analysis (Zhao Z. et al., 2009, Zhou and Rempfer, 2013).

\section{RESULTS OF RESEARCH}

As a result of the numerical simulation, flow through the single windmill ring was obtained, for which the velocity distribution was included in Figure 3. From the obtained results, as expected, we observe an increase in velocity on both sides of the windmill, and aerodynamic shadow behind the windmill, as well as variable speed distribution in rotor zone. The air flow entering into the rotor rotating zone obtains a vortex in the opposite direction to the rotor.

Figure 4 shows a graph of pressure change along the $x$-axis passing through the center of the rotor (coordinate $x=0$ ), from the output (coordinate $x=-6$ ) to the input $(x$-coordinate $=6$ ). From the course of variationwe you can see a 
gradual change in pressure in the zone before the windmill in relation to the pressure inside the rotor, with another step drop in pressure behind the turbine, which in some way will reflect the transfer of energy from the wind stream to the windmill's rotor.

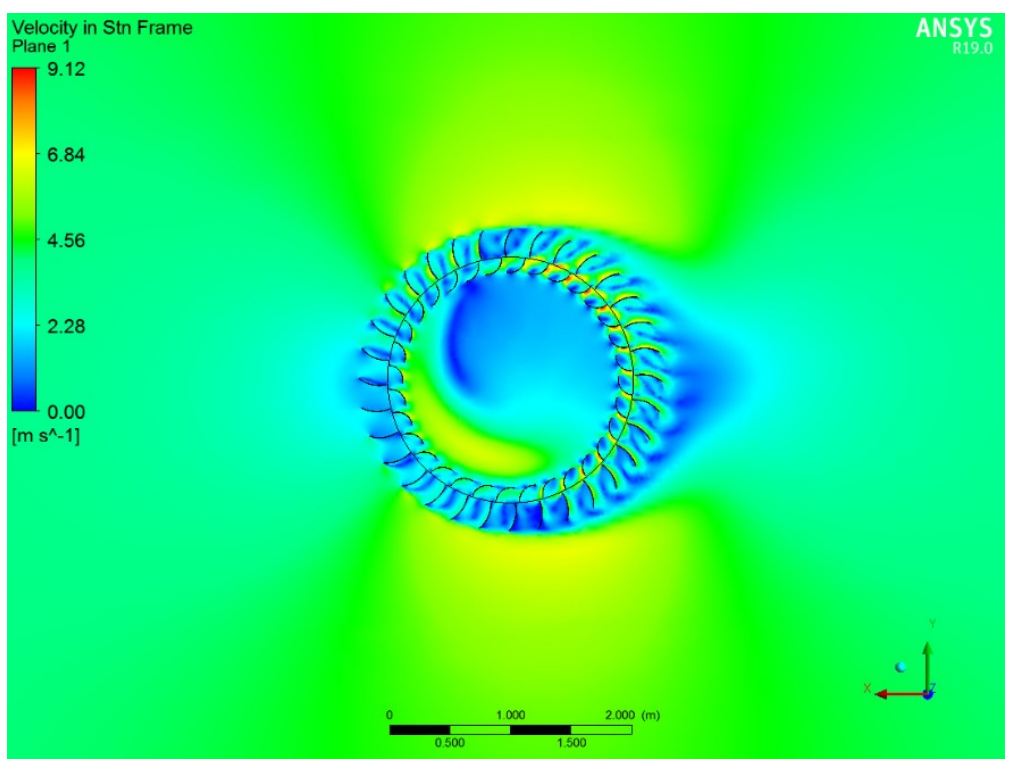

Fig. 3 Velocity distribution

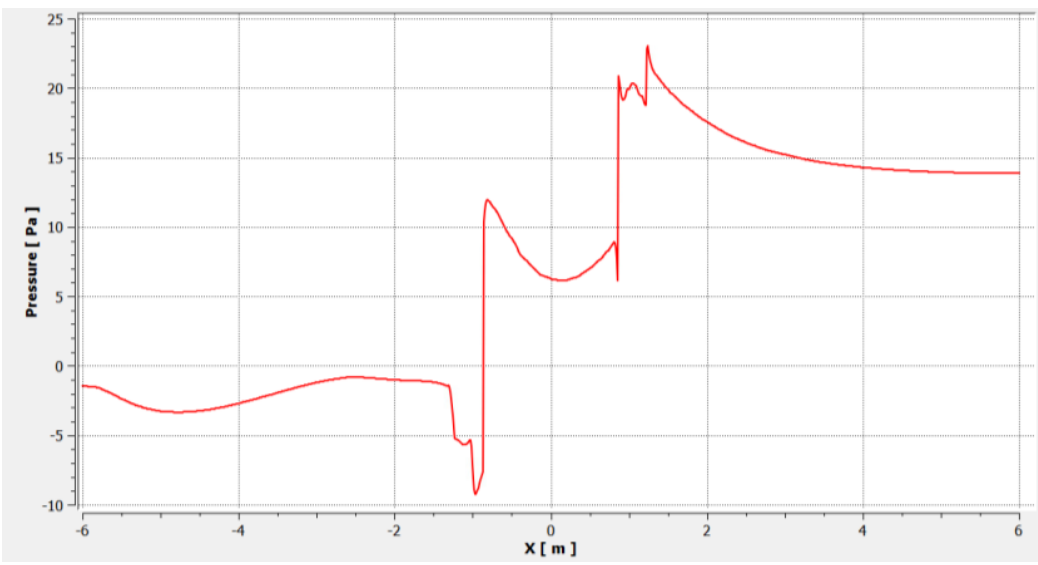

Fig. 4 Pressure change along the $x$-axis passing through the center of the rotor, from the output to the input of domain

Furthermore, Figure 5 presents the pressure distribution on the surface of the rotor blades and air guides. From which we see a variable distribution of pressure values for individual blades on the perimeter of both the rotor and the blades. The value of the pressure on the blades on the input side is at the level of $30 \mathrm{~Pa}$, while on the blades on the exit side at the level of $-40 \mathrm{~Pa}$.

On the basis of the obtained result, it can be concluded that the driving force of the rotor will be the effect of both the hydrodynamic air stream on the blades as well as the force generated due to the pressure difference resulting from the shape of the rotor blade. For the conducted analysis, the torque acting on the rotor is $10.7 \mathrm{Nm}$, which, after taking into account the rotational speed of the rotor, gives a turbine power of $\mathrm{Po}=17.12$ Watt. 


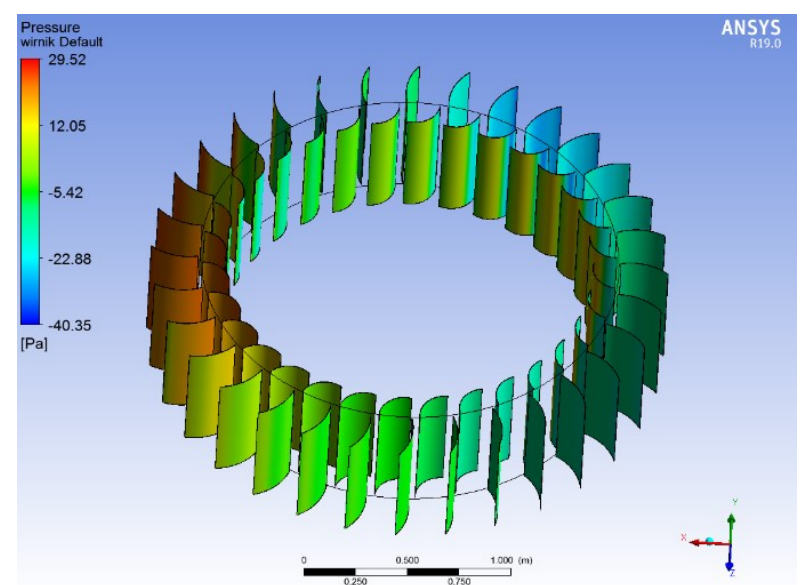

Fig. 5 Pressure distribution over rotor blades and air guides

For a given structure, assuming a wind speed of $4 \mathrm{~m} / \mathrm{s}$, taking into account the vertical cross section of the wind turbine single stage, the theoretical wind stream power calculated on the basis of equation (1) will be 47.51 Watt.

$$
P_{w}=\frac{1}{2} * \rho * A * v^{3}
$$

As a result, based on the power of the turbine and the power of the wind stream, we can determine the power factor for the analyzed structure, which on the basis of the dependence (2) will be $C_{p r}=0.36$.

$$
C_{p r}=\frac{P_{o}}{P_{w}}
$$

The analysis shows that the analyzed turbine has a higher Betz power factor than typical designs of wind turbines with a vertical axis, for which the power factor value usually does not exceed $25 \%$, which in effect gives greater possibilities in the use of theoretical wind power.

\section{ANALYSIS OF THE INFLUENCE OF SHAPING AND PLACING OF BLADES ON THE POWER OBTAINED}

In the preliminary simulations, were also carried out test analyzes to verify the level of influence on the generated power of both the arrangement of the rotor blade as well as its shape. The basic simulation was performed for the rotor blade shown in Figure 6, the chord of which was set at an angle of $45^{\circ}$ to the tangent line to the rotor at the point of contact between the blade and the rotor edge. In order to compare the effect of the blade position on the obtained power, numerical simulations were performed for the blade angle setting, initially reducing the angle to $35^{\circ}$, and then increasing it to $55^{\circ}, 65^{\circ}$ and $75^{\circ}$, respectively.

The results of the analyzes carried out for the angle of the rotor blade in the range $35^{\circ}-75^{\circ}$, while maintaining the other parameters of the analysis are given in Table 1. The results clearly show that the setting of the rotor blade as expected influences the generated torque and power, received with assumed angle gradation maximum for $55^{\circ}$. 
Fig. 6 Geometrical parameters of blades modified during simulation

Table 1 Torque and power value obtained in numerical simulation for various setting of rotors blade

\begin{tabular}{|c|c|c|}
\hline Blade angle [ ${ }^{\circ}$ ] & Torque [Nm] & Power [W] \\
\hline 35 & 10.1 & 16.1 \\
\hline 45 & 10.7 & 17.1 \\
\hline 55 & 12.2 & 19.4 \\
\hline 65 & 9,5 & 15.1 \\
\hline 75 & 7.9 & 12.6 \\
\hline
\end{tabular}

An analysis was also carried out for three values of the bending radius of the rotor blade (Fig. 6) respectively $110 \mathrm{~mm}, 125 \mathrm{~mm}$ and $140 \mathrm{~mm}$. The obtained result is shown in Table 2.

Table 2 Torque and Power values obtained In numerical simulations for various bending radius of rotors blade

\begin{tabular}{|c|c|c|}
\hline Blade radius [mm] & Torque [Nm] & Power [W] \\
\hline 110 & 5.57 & 8.92 \\
\hline 125 & 10.70 & 17.12 \\
\hline 140 & 6.24 & 9.99 \\
\hline
\end{tabular}

Obtained results confirm impact of blades radius on delivered power. Maximum value of power was obtained for blade with radius $125 \mathrm{~mm}$. Based on data in Table 1 and 2 we can tell that greater influence on harvested energy will have the shape of blades than its setting.

\section{CONCLUSION}

Preliminary analysis of the work of a wind turbine with a vertical axis of rotation with air guides confirms the possibility of using such a structural solution as a device for obtaining energy from the wind. The conducted analyzes indicate the possibility of obtaining a higher power factor for structures with air guides than in the classical solutions of this type. At the same time, the obtained Betz power factor is still significantly lower than the possible coefficient in structures with the horizontal axis. Nevertheless, as shown by the conducted analyzes, it is possible to use numerical simulation to optimize the structure as well as the operating parameters of the analyzed turbine. This allows to hope for obtaining a higher power factor, which in effect would significantly increase the profitability of using this type of turbine in practice.

At the same time, it should be stated here that these are preliminary analyzes, which give a very general information about the work of a vertical axis turbine with air guides, however, allow to state that the topic is attractive enough and 
the initial results so promising that the optimization studies of mentioned above constructions are justified and may be the subject of further work.

\title{
REFERENCES
}

Hudy W., Piaskowska-Silarska M., Noga H., Kulinowski W., Pytel K., Gumuła S., (2018). Analysis of the possibility of reducing the amount of air pollution using photovoltaic systems. ICCC'2018, Hungary, May 28-31

TURLEJ T., BANAŚ M. (2017). Management of communal sewage waste in Poland / // W: SGEM 2017: 17-th international multidisciplinary scientific geoconference: ecology, economics, education and legislation: 29 June-5 July, 2017, Albena, Bulgaria : conference proceedings. Vol. 17 iss. 51, pp. 627-634.

Hudy W., Pytel K., Marikutsa U., Piaskowska-Silarska M., Gumuła S., Farmaha I., (2019). Application of evolutionary algorithms to analysis the possibilities of wind energy use. W: ICCC 2019. Kraków - Wieliczka, May 26-29, 2019 e-ISBN: 9781-7281-0701-1. pp. 1-7.

Rahman M., Salyers T.E., El-Shahat A., Ilie M., Ahmed M., Soloiu V. (2018). Numerical and Experimental Investigation of Aerodynamic Performance of Vertical-Axis Wind Turbine Models with Various Blade Designs. Journal of Power and Energy Engineering, 6, 26-63.

Kacprzak K., Sobczak K. (2013). Numeryczna analiza przepływu przez turbinę Savoniusa. Turbomachinery no. 143.

Fujisawa N. and Gotoh F. (1994), Experimental study on the aerodynamic performance of a Savonius rotor. Journal of Solar Energy Engineering, 116(3): 148-152.

Spera D.A.(2009). Wind turbine technology: fundamental concepts of wind turbine engineering. ASME Press.

Wagner H.J. and Mathur J., (2009). Technology and operation. Green energy and technology. Springer.

Zhao Z. and Zheng and Y. Xu, X. (2009). Research on the improvement of the performance of savonius rotor based on numerical study. In Sustainable Power Generation and Supply, 2009. SUPERGEN '09. International Conference on, pp. 1-6.

Zhou T. and Rempfer D. (2013). Numerical study of detailed flow field and performance of Savonius wind turbines. Renewable Energy, 51(0): pp. 373-381.

\begin{abstract}
.
Currently, obtaining energy from renewable sources, as never before, it has become one of the most important areas, but at the same time challenges in human activities. This is the effect of many factors simultaneously, including because of the media trumpeted the greenhouse effect, the necessity of reducing $\mathrm{CO}_{2}$ emissions, shrinking natural resources, and thus the desire to protect their welfare including conservation (including natural resources) for future generations. The paper presents the results of preliminary numerical analyzes of work for vertical axis wind turbines equipped with air guides. The carried out analyzes indicate the potential possibility of using this type of construction in practice. The obtained power factor value is higher than in classic wind turbines with a vertical axis. The conducted analyzes also indicate the possibility of optimizing the proposed constructional solution in order to increase efficiency of obtaining energy from the wind.
\end{abstract}

Keywords: Vertical axis wind turbine, numerical modeling 